\title{
Imaging polarimetry and spectropolarimetry of comet C/2013 R1 (LoveJoy)
}

\author{
Galin Borisov ${ }^{\mathrm{a}, \mathrm{b}, *}$, Stefano Bagnulo ${ }^{\mathrm{b}}$, Plamen Nikolov ${ }^{\mathrm{a}}$, Tanyu Bonev ${ }^{\mathrm{a}}$ \\ ${ }^{a}$ Institute of Astronomy and National Astronomical Observatory, Bulgarian Academy of Sciences, 72, Tsarigradsko Chaussee Blvd., BG-1784 Sofia, Bulgaria \\ ${ }^{b}$ Armagh Observatory, College Hill, Armagh BT61 9DG, Northern Ireland, UK
}

\begin{abstract}
We have obtained imaging polarimetry of the comet C/2013 R1 (Lovejoy) with 2-Channel-Focal-Reducer Rozhen instrument at $2 \mathrm{~m}$ Ritchey-Chrétien-Coudé telescope of the Bulgarian National Astronomical Observatory Rozhen in two dust continuum filters covering wavelength intervals clear from molecular emissions and centred at $4430 \AA$ in (blue filter) and at $6840 \AA$ in (red filter). In imaging mode we measured the degree of linear polarisation $17.01 \pm 0.09 \%$ in the blue and $18.81 \pm 0.02 \%$ in the red, which is in a very good agreement with measurements of other comets at the similar phase angle. We have also obtained polarisation maps in both filters. We found a strong correlation between the spatial distribution of the polarisation and the dust colour. Spectropolarimetry of the nucleus region shows an increase of the polarisation with wavelength, and a depolarisation in the spectral regions with gas emission lines, most noticeable in $\mathrm{C}_{2}$ emission band, which shows a polarisation of $6.0 \pm 1.1 \%$.
\end{abstract}

Keywords: comets, C/2013 R1 (Lovejoy), polarimetry, spectropolarimetry, dust, molecules

\section{Introduction}

Polarimetry is sensitive to the physical properties of the dust particles: size, shape, porosity, orientation and chemical composition represented by its material complex refractive index. Polarimetric measurements give us the possibility to determine some parameters that cannot be determined trough traditional intensity measurements.

The first polarimetric observations of comets were made by François Arago (1854), who discovered the polarised light in the Great Comet 1819 II.

Later, observations of comets clarified some common characteristics of the polarised light, for example that usually the plane of polarisation is perpendicular to the scattering plane, and that there are variation of the polarisation in different parts of the comet (coma, tail). Contemporary polarimetric observations of comets began with the work of Yngve Öhman (1939. 1941), who observed for the first time the continuum polarisation in comets and the polarisation of the emission lines.

Most of the recent polarimetric observations of comets have been obtained by Kiselev and collaborators. Kiselev et al. (2005) has also created a database with more than 2600 measurements of linear and circular polarisation for 64 comets since 1940s.

Most of the polarimetric observations of small Solar system bodies are aimed at measuring the variation of the polarisation with phase angle (which actually is $180^{\circ}$ - scattering angle) and also its dependence on wavelength.

\footnotetext{
Based on data collected with $2 \mathrm{~m}$ RCC telescope at Rozhen National Astronomical Observatory.

${ }^{*}$ Corresponding author

Email address: gborisov@astro.bas.bg (Galin Borisov)
}

From the theoretical side, many works have been carried out by Kolokolova and collaborators (Kolokolova et al., 1997, Kolokolova and Jockers, 1997; Kolokolova et al., 2004).

The polarisation of the dust jet-like structures in the dust coma of the comet Hale-Bopp was obtained for the first time by Hadamcik et al. (1997) and was discussed later on in Hadamcik and Levasseur-Regourd (2003).

A recent review of all comets investigation can be found in the books by Mishchenko et al. (2010) and Kiselev et al.(2015).

Comet C/2013 R1 (Lovejoy) was discovered by Terry Lovejoy (Thornlands, Queensland, Australia) with images acquired on 2013 September 7 and 8, using his 20-cm reflector and a CCD camera.

Other polarimetric measurements of the comet C/2013 R1 (Lovejoy) are presented by Furusho et al. (2014) (imagine polarisation with the Subaru telescope) and by Rosenbush et al. (2014) (linear and circular polarimetric measurements and their modelling).

\section{Observations}

Comet C/2013 R1 (Lovejoy) was observed during a multiinstrument campaign with the $2 \mathrm{~m}$ Ritchey-Chrétien-Coudé (RCC) telescope of the Bulgarian National Astronomical Observatory (BNAO) Rozhen from 20 Dec 2013 until 07 Jan 2014. Because of the target brightness we could achieve a relatively high $\mathrm{S} / \mathrm{N}$ ratio and obtain high accuracy of polarimetric measurements. C/2013 R1 (Lovejoy) was a new comet which approach to the inner Solar System for the first time and giving us an opportunity to investigate the pristine material from the era of the Solar System formation. 


\subsection{Instrumentation}

Polarimetric observations were performed with the 2Channel-Focal-Reducer Rozhen (FoReRo2) (Jockers et al. 2000) attached at the Cassegrain focus of the $2 \mathrm{~m}$ RCC telescope. In polarimetric mode, FoReRo 2 is equipped with a Wollaston prism, placed before a dichroic beam splitter, which splits the signals into two different channels, allowing us to reconstruct polarimetric maps of extended objects in two spectral regions simultaneously, using narrow band filters. By replacing the filters with two grisms, we can perform spectropolarimetric measurements. An example of raw spectropolarimetric image can be seen in Fig. 1 .

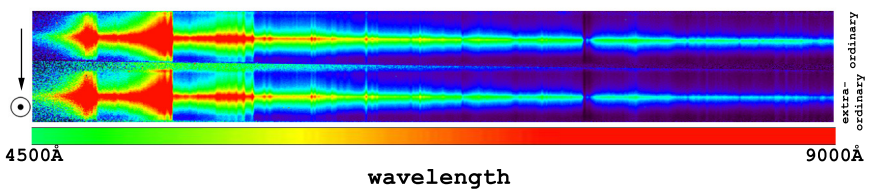

Figure 1: Spectropolarimetric image of comet C/2013 R1 (Lovejoy).

Imaging polarimetry was obtained in two dust continuum filters covering wavelength intervals clear from molecular emission and centred at $4430 \AA$ and $6840 \AA$, having a passband of $35 \AA$, and $71 \AA$ and hereafter called IF443 and IF684, respectively (see Fig. 2).

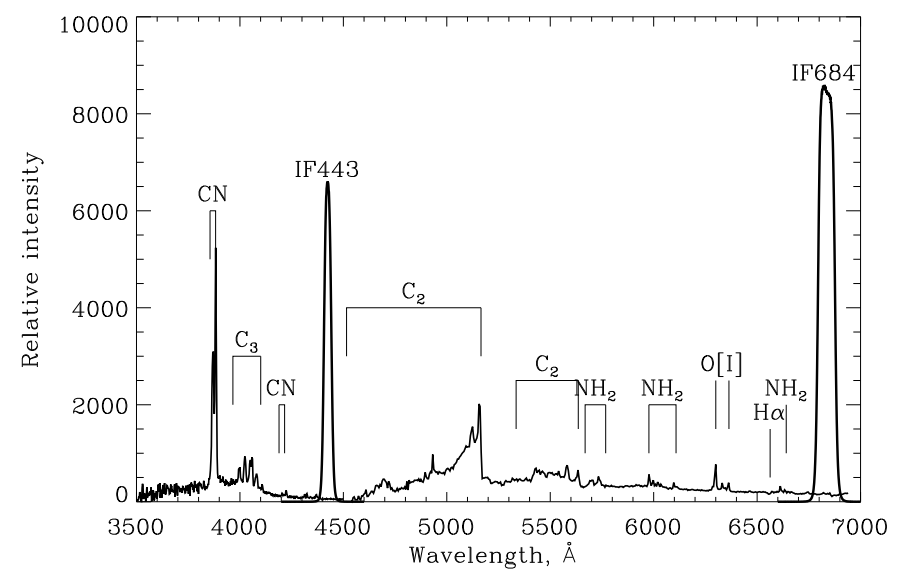

Figure 2: Continuum filters transmission curves (IF443 \& IF684) overplotted on a comet spectrum

\subsection{Comet C/2013 R1 (Lovejoy)}

Imaging and spectropolarimetric data were obtained on December 29 and January 3 respectively, with FoReRo2. The geometrical conditions during the observations are shown in table 1.

\subsection{Data Reduction}

All images were pre-processed through a standard bias subtraction and flat field correction.

\begin{tabular}{|c|c|c|c|c|}
\hline Date & $1^{a}, \mathrm{AU}$ & $4^{b} \mathrm{AU}$ & $a^{c}$ deg & Obs. mode \\
\hline 20 Dec 2013 & 0.8132 & 0.8562 & 72.2 & HRS $^{d}$ \\
\hline 21 Dec 2013 & 0.8123 & 0.8765 & 71.1 & HRS \\
\hline 22 Dec 2013 & 0.8118 & 0.8965 & 70.1 & HRS \\
\hline 23 Dec 2013 & 0.8118 & 0.9161 & 69.1 & HRS \\
\hline 24 Dec 2013 & 0.8122 & 0.9355 & 68.1 & HRS \\
\hline 29 Dec 2013 & 0.8210 & 1.0305 & 63.0 & $\operatorname{ImPo} e^{e}$ \\
\hline 30 Dec 2013 & 0.8240 & 1.0495 & 62.0 & $\mathrm{NBF}^{\mathrm{L}}$ \\
\hline 31 Dec 2013 & 0.8275 & 1.0675 & 61.0 & $\mathrm{H}_{2} \mathrm{O}^{+}$ \\
\hline 03 Jan 2014 & 0.8405 & 1.1205 & 58.1 & SPo $g$ \\
\hline 08 Jan 2014 & 0.8631 & 1.1865 & 54.6 & $\mathrm{NBF} \& \mathrm{H}_{2} \mathrm{O}^{+}$ \\
\hline
\end{tabular}

Table 1: Observing Log

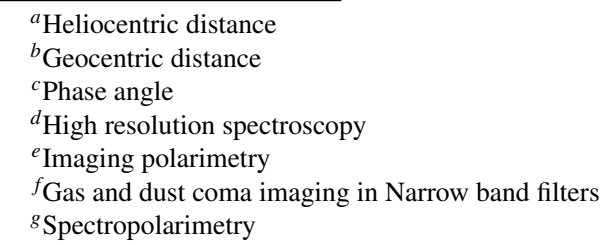

At the time of our observations, the polarimetric optics of FoReRo2 included a Wollaston prism but not a retarder waveplate, preventing us from adopting a beam-swapping technique to minimise instrumental effects (see, e.g. Bagnulo et al., 2009). Previous experience showed that polarimetric observations with FoReRo 2 were affected by non-negligible and non-constant instrumental polarisation. In an attempt to mitigate this problem, we decided to obtain observations at two instrument position angles, one with the principal plan of the Wollaston prism aligned to the scattering plan (i.e., the plan defined by the sun, the comet and the observer) and one perpendicular to it. By denoting with $f^{\|}$and $f^{\perp}$ the fluxes in the parallel and in the perpendicular beams, and with $k_{\|}$and $k_{\perp}$ the transmission functions in the parallel and in the perpendicular beam of the Wollaston prism respectively, the observed quantity is

$$
\begin{aligned}
\frac{\hat{Q}}{I} & =\frac{1}{2}\left[\left(\frac{f^{\|}-f^{\perp}}{f^{\|}+f^{\perp}}\right)_{\mathrm{PA}=\phi+90^{\circ}}-\left(\frac{f^{\|}-f^{\perp}}{f^{\|}+f^{\perp}}\right)_{\mathrm{PA}=\phi}\right] \\
& =\frac{1}{2}\left[\frac{k_{\|}(I+Q)-k_{\perp}(I-Q)}{k_{\|}(I+Q)+k_{\perp}(I-Q)}-\frac{k_{\|}(I-Q)-k_{\perp}(I+Q)}{k_{\|}(I-Q)+k_{\perp}(I+Q)}\right] \\
& =\frac{\left(k_{\|}+k_{\perp}\right)^{2} I Q-\left(k_{\|}-k_{\perp}\right)^{2} I Q}{\left(k_{\|}+k_{\perp}\right)^{2} I^{2}-\left(k_{\|}-k_{\perp}\right)^{2} Q^{2}}
\end{aligned}
$$

where $\phi$ is the angle is the angle between the direction ObjectNorth Pole and the direction Object-Sun. If $k_{\|} \simeq k_{\perp}$ we obtain

$$
\frac{Q}{I} \simeq \frac{\hat{Q}}{I} .
$$

where $Q / I$ is the reduced Stokes parameter $Q$ measured assuming as a reference direction that one perpendicular to the scattering plane. We did not measure $U / I$, assuming that for symmetric reasons it is probably zero. Of course, the images were combined together after a 90 degrees rotation. 


\section{Results}

\subsection{Aperture polarimetry}

The aperture photometry with a circular aperture with a radius of $13 \times 10^{3} \mathrm{~km}$ on the comet was performed to measure the intensity of the two orthogonal polarised beams $f^{\|}$and $f^{\perp}$. The background was measured in the farthest possible place in the sunwards direction. The results for both continuum filter IF443 and IF684 are $P_{443}=17.01 \pm 0.09 \%$ and $P_{684}=18.81 \pm 0.02 \%$ respectively. Their comparison with the Kiselev database (Kiselev et al., 2005) is shown in Fig. 3 and is in a good agreement with the data for the comet 1P/Halley at similar phase angles $\left(65^{\circ}\right)$.

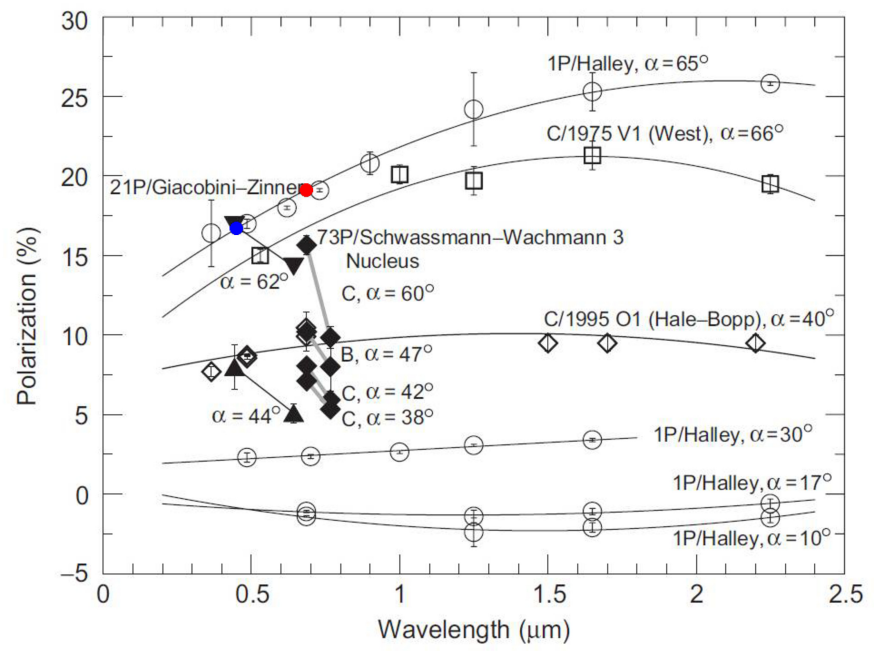

Figure 3: Comparison of polarimetric measurement of comet C/2013 R1 (Lovejoy) and Kiselev database (Kiselev et al. 2005) (error bars of our measurements are smaller than the symbols)

\subsection{Imaging polarimetry}

In order to investigate the spatial distribution of the linear polarisation of the light reflected from cometary dust we performed imaging polarimetry of the comet's coma.

We construct polarisation maps by calculating the degree of linear polarisation, using the beam swapping technique, for each pixel in a pre-selected region around comet photo centre. The resultant polarimetric maps and radial profiles for the dust continuum filter IF684 are presented in the top and bottom panel of Fig. 4 , respectively.

Due to the low signal-to-noise ratio, the polarimetric map in IF443 dose not display any remarkable structures. By contrast, the polarisation map in IF684 shows clearly a highly polarised structure apart from the nucleus.

The polarisation degree in a square region $11 \times 1110^{3} \mathrm{~km}$ enveloped this structure is slightly higher than that of the total coma, i.e. $P_{684}^{\text {jet }}=18.83 \pm 0.045 \%$

In order to investigate the nature of this structure, we first try to connect this highly polarised structure with a jet-like structure in the dust coma. Therefore we used an enhanced procedure to reveal any inhomogeneity around the nucleus caused by
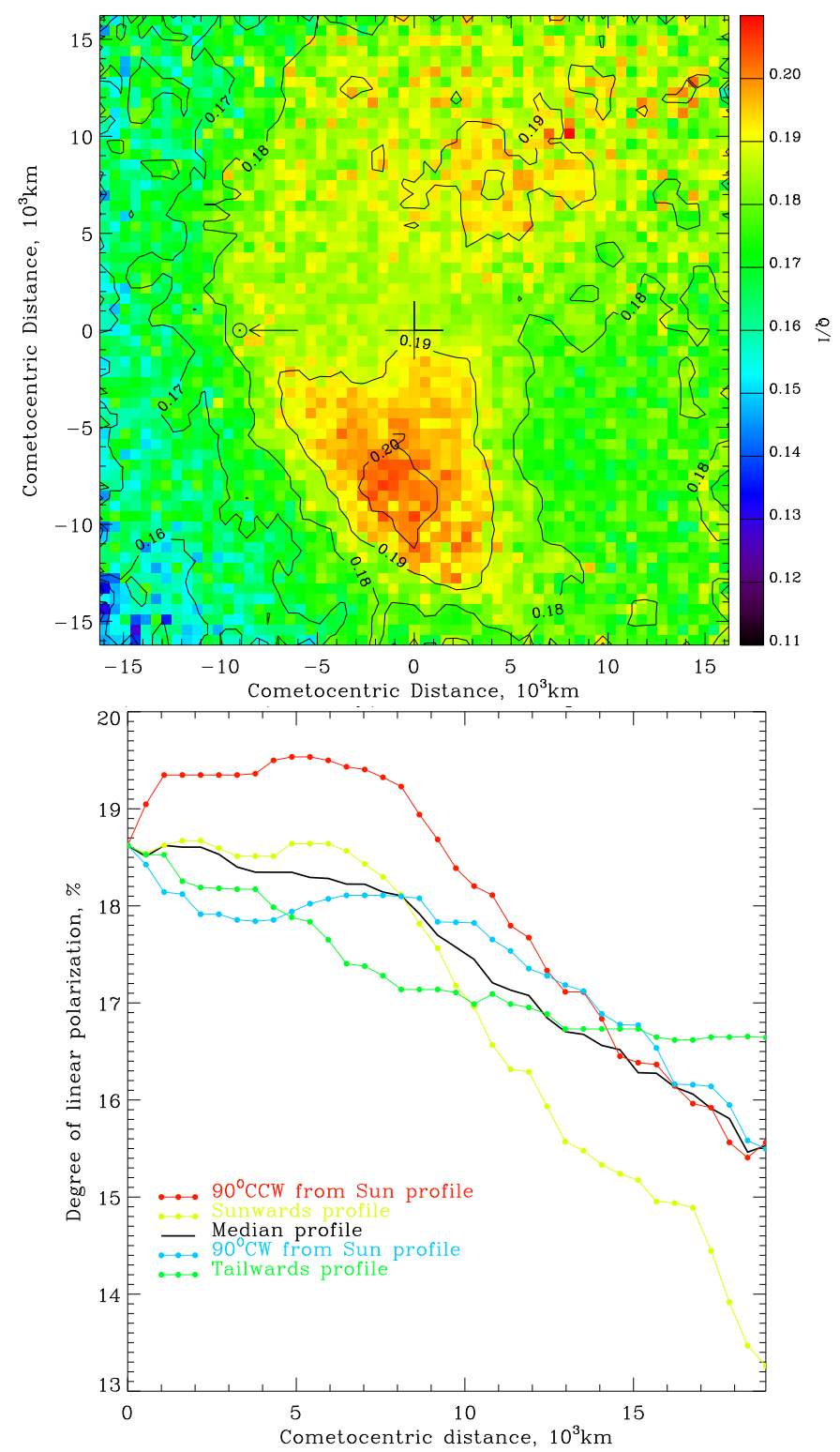

Figure 4: Polarisation map with a marked comet photocentre (+) and direction to the Sun $(\odot \leftarrow)$ (top panel) and radial profiles in different directions (bottom panel) in IF684

unsteady outflow from the nucleus. This procedure remove the average coma, modelled by fitting the radial profiles of the dust at different azimuths with a power low. Afterwards this model was subtracted and only the jet-like structure remains in the image. In the top panel of Fig. 5, such a structure is clearly visible, but it is shifted from the polarisation one, presented with overplotted contours.

Next, we compared it with the distribution of the dust normalised reflectivity gradient, or so called reddening. That is why we construct the so called colour map of the dust by calculating the quantity 

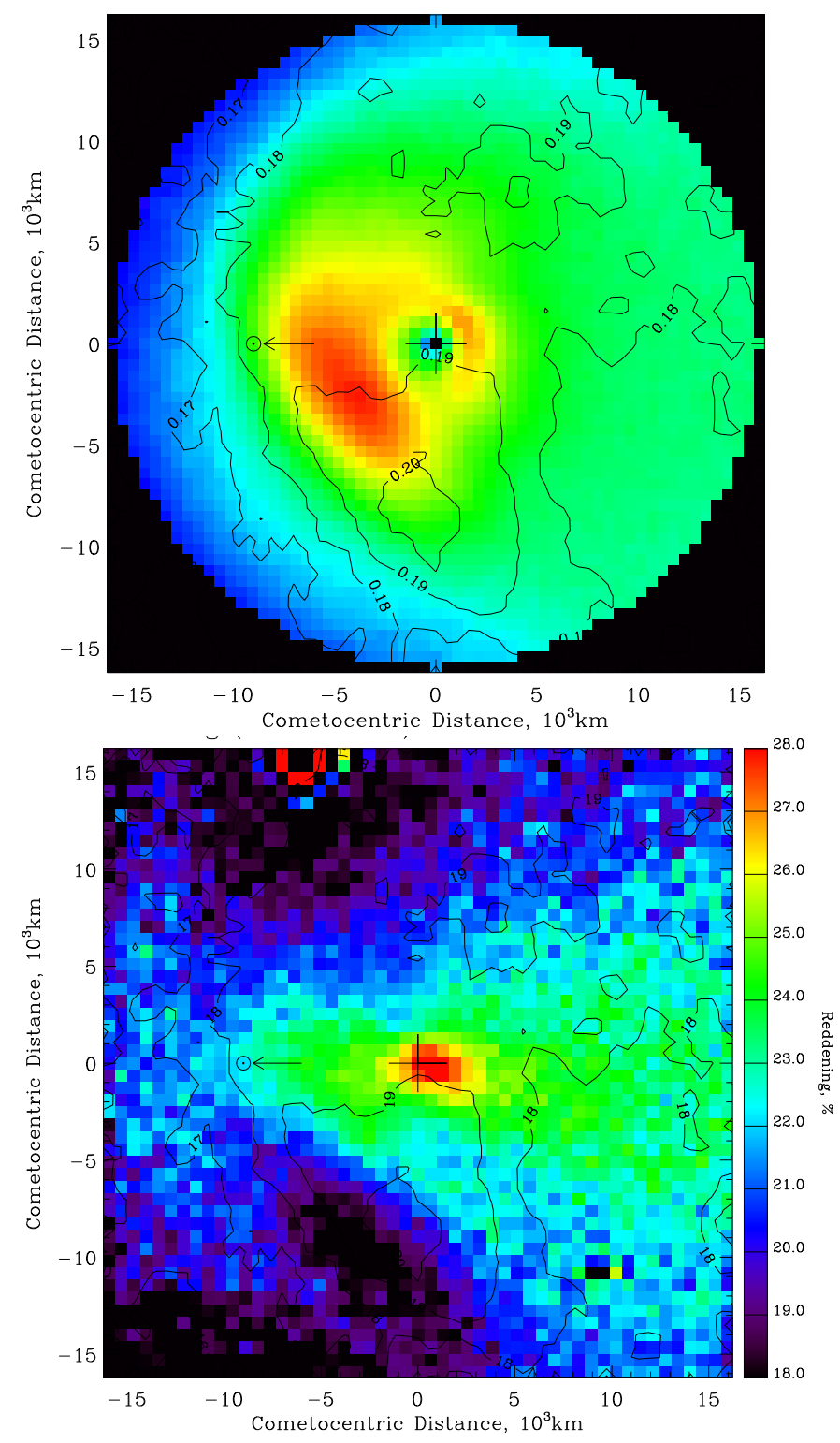

Figure 5: Enhanced coma structure in IF684 (top panel) and colour map (bottom panel) compared with polarisation (overplayed contours)

$$
S^{\prime}\left(\lambda_{1}, \lambda_{2}\right)=\frac{1}{\bar{S}} \frac{\partial S}{\partial \lambda}
$$

where $\bar{S}=\left(S_{\lambda_{2}}-S_{\lambda_{1}}\right) / 2$ and is usually expressed in $\% / 1000 \AA$, for each pixel in both dust continuum images in IF443 and IF684. The resultant colour map is presented in the bottom panel of figure 5. The connection between high polarisation and low reddening is clearly visible. The interpretation is that low reddening means small particles, or very dark ones, both of which give high polarisation.

\subsection{Spectropolarimetry}

Polarisation spectrum of the comet C/2013 R1 (Lovejoy) is shown in Fig. 6 .

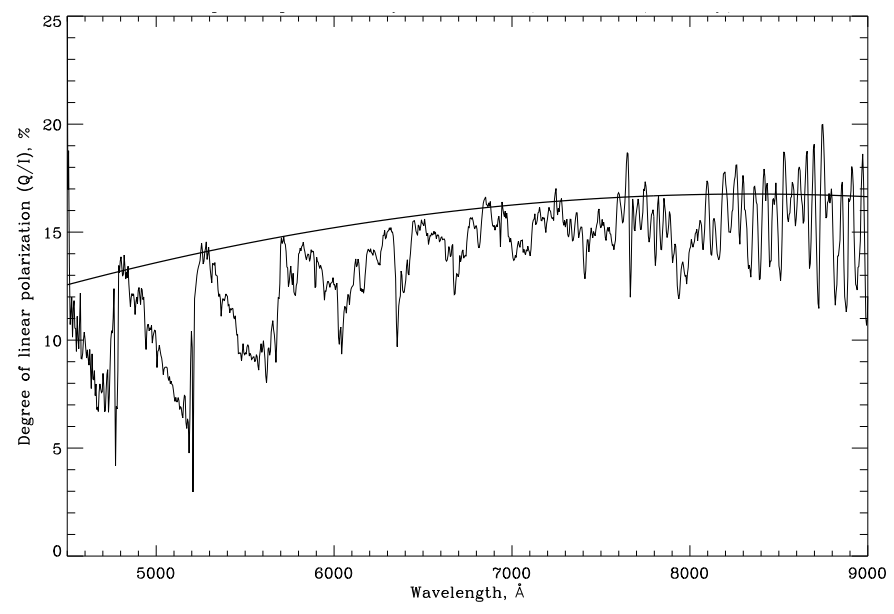

Figure 6: Spectropolarisation of comet C/2013 R1 (Lovejoy) and a fitted continuum polarisation.

We carried out the the polynomial fit to the dust continuum polarisation. The result for comet $1 \mathrm{P} /$ Halley at different phase angles are also presented (Kiselev et al. 2005) and our observations are very well fitted to the phase angle and wavelength trend of the comet 1/P Halley (see Fig. 7).

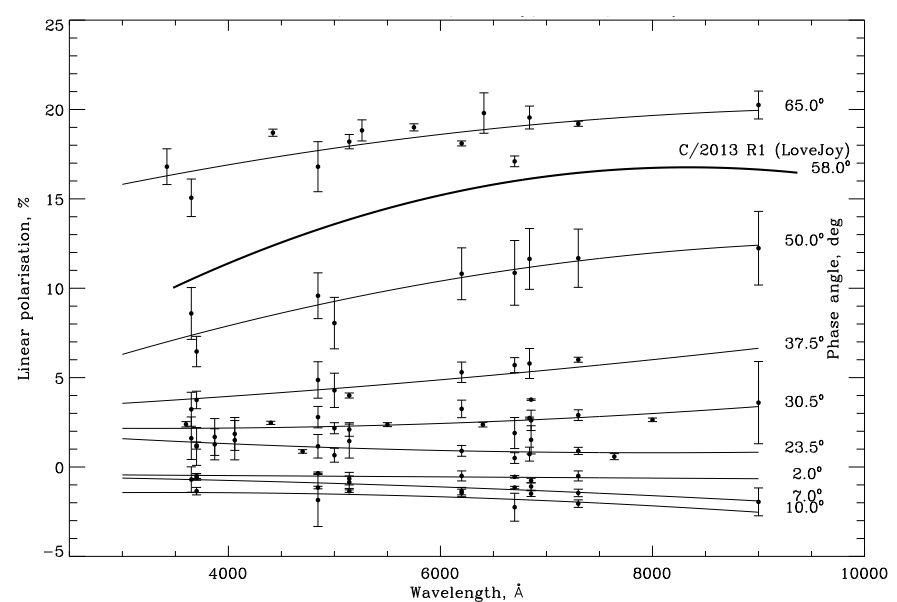

Figure 7: Comparison of the comet C/2013 R1 (LoveJoy) with the typical comet $1 \mathrm{P} /$ Halley measurements at different phase angles.

The polarisation in molecular spectral lines is lower than that in the continuum. According to the theory of diatomic molecules it should be a constant of 1/7 (Feofilov, 1961). To estimate the polarisation of the emission lines we first subtract the continuum contribution from both spectra in the ordinary and extraordinary beams. Then, using the remaining flux from resonance fluorescence of the molecules, we calculate the degree of linear polarisation following the procedure described in section 2.3 for each wavelength bin. The result for the main $\mathrm{C}_{2}$ molecular band is presented in Fig. 8

The average value of linear polarisation over the whole molecular band of $\mathrm{C}_{2}$ is $6.0 \pm 1.1 \%$. This value is less than half that of the theoretical one, which is based on theory of 


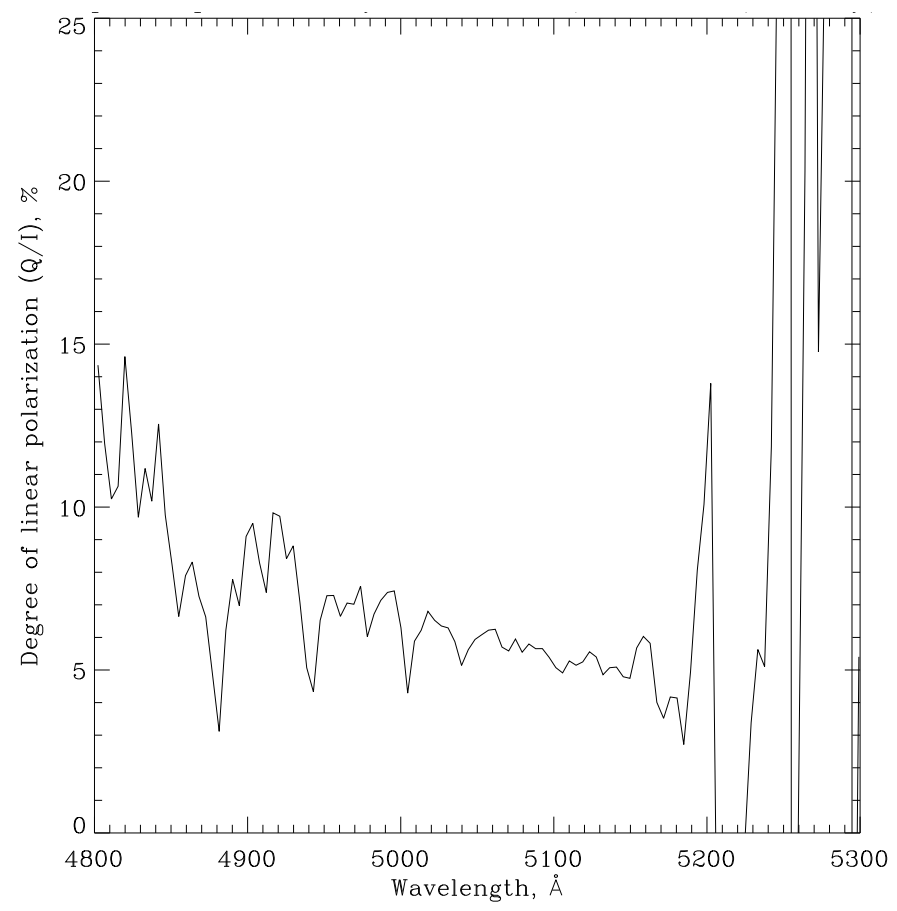

Figure 8: Polarisation spectrum of the $\mathrm{C}_{2}$ emission line in the coma of the comet C/2013 R1 (LoveJoy)

anisotropic rotating oscillators. Also these values are for pure gas without the presence of other species. The theory says that polarisation of the fluorescence of diatomic molecules is reduced when foreign gases are introduced, and collisions occur, resulting a depolarisation. Therefore we conclude that this lower value of linear polarisation in the $\mathrm{C}_{2}$ molecular band is due to collisions with other species in the comet's coma.

The excitation by unidirectional natural radiation leading to linear polarisation of an emission line has a maximum polarisation value for a phase angle of $90^{\circ}$. The expected variation of gas polarisation with phase angle $\alpha$ is given by the expression:

$$
P(\alpha)=\frac{P_{\max } \sin ^{2} \alpha}{1+P_{\max } \cos ^{2} \alpha}
$$

From our measurements we calculate $\mathrm{P}_{\max }=8.5_{-1.6}^{+2.5} \%$. $\mathrm{Kr}$ ishna Swamy (2010) says that the theoretical value of $\mathrm{P}_{\max }$ for the $\mathrm{C}_{2}$ and $\mathrm{CN}$ molecules is $7.7 \%$, which is within our confidence interval $6.9-11.2$.

\section{Conclusions}

- We have measured the degree of linear polarisation in the continuum filters IF443 and IF684 of the dust ejected from comet $\mathrm{C} / 2013 \mathrm{R} 1$ (Lovejoy), and our results $\left(P_{443}=\right.$ $17.01 \pm 0.09 \%$ and $\left.P_{684}=18.81 \pm 0.02 \%\right)$ are in a good agreement with measurements of the typical comet 1P/Halley..

- We have investigated the spatial distribution of the dust in both continuum filters. A polarisation feature in the red was found and compared with jet-like structures in the dust coma, and connected with low values in the map of the dust colour, which shows that this region is populated rather with small or very dark grains.

- Furusho et al. (2014) presented imaging polarisation of the comet Lovejoy with the Subaru telescope, and their results (including the detection of coma features with high polarisation values) are consistent with our results.

- We have obtained a spectropolarimetry of the comet and found the change of the degree of linear polarisation of the dust with wavelength. This is in a good agreement with the results obtained for comet $1 \mathrm{P} /$ Halley.

- In the $\mathrm{C}_{2}$ molecule emission band we have measured $6.0 \pm 1.1 \%$ degree of linear polarisation. The results deviate from the theoretical value for diatomic molecules $14.3 \%$ (Feofilov, 1961), but are in good agreement with a value measured for other comets $\mathrm{P}_{\max }=7.7 \%$ (Krishna Swamy, 2010). This can be used to explain the depolarisation effect in the molecular coma.

\section{Acknowledgements}

The authors gratefully acknowledge the observing grant support from the Institute of Astronomy and Rozhen National Astronomical Observatory, Bulgarian Academy of Sciences.

GB and SB also gratefully acknowledge financial support from the COST Action MP1104 "Polarisation as a tool to study the Solar System and beyond".

GB also gratefully acknowledge financial support from the Federation of Finnish Learned Societies and the organisers of the ACM2014.

PN acknowledge financial support from ESF and Bulgarian Ministry of Education and Science under the contract BG051PO001-3.3.06-0047.

\section{References}

Arago, F., 1854. Astronomie populaire. Gide et Baudry, Paris.

Bagnulo, S., Landolfi, M., Landstreet, J. D., Landi Degl'Innocenti, E., Fossati, L., Sterzik, M., Sep. 2009. Stellar Spectropolarimetry with Retarder Waveplate and Beam Splitter Devices. PASP 121, 993-1015.

Feofilov, P., 1961. The Physical Basis of Polarized Emission. Consultants Bureau, New York.

Furusho, R., Terai, T., Shinoda, T., Watanabe, J., Jul. 2014. C/2012 S1 (ISON), C/2013 R1 (Lovejoy), and updates of the imaging polarimetric survey. In: Muinonen, K., Penttilä, A., Granvik, M., Virkki, A., Fedorets, G., Wilkman, O., Kohout, T. (Eds.), Asteroids, Comets, Meteors 2014. p. 173.

Hadamcik, E., Levasseur-Regourd, A. C., May 2003. Dust evolution of comet C/1995 O1 (Hale-Bopp) by imaging polarimetric observations. A\&A 403, $757-768$.

Hadamcik, E., Levassuer-Regourd, A. C., Renard, J. B., Jul. 1997. Ccd Polarimetric Imaging Of Comet Hale-Bopp (C/1995 O1). Earth Moon and Planets 78, 365-371.

Jockers, K., Credner, T., Bonev, T., Kisele, V. N., Korsun, P., Kulyk, I., Rosenbush, V., Andrienko, A., Karpov, N., Sergeev, A., Tarady, V., Sep. 2000. Exploration of the solar system with the Two-Channel Focal Reducer at the $2 \mathrm{~m}$ RCC telescope of Pik Terskol Observatory. Kinematika i Fizika Nebesnykh Tel Supplement 3, 13-18. 
Kiselev, N., Rosenbush, V., Jockers, K., Velichko, S., Kikuchi, S., Dec. 2005. Database of Comet Polarimetry: Analysis and Some Results. Earth Moon and Planets 97, 365-378.

Kiselev, N., Rosenbush, V., Levasseur-Regourd, A.-C., Kolokolova, L., 2015. Comets. Cambridge University Press, Ch. 22, pp. 379-404.

Kolokolova, L., Hanner, M. S., Levasseur-Regourd, A.-C., Gustafson, B. Å. S., 2004. Physical properties of cometary dust from light scattering and thermal emission. University of Arizona Press, Ch. VI, pp. 577-604.

Kolokolova, L., Jockers, K., Dec. 1997. Composition of cometary dust from polarization spectra. P\&SS 45, 1543-1550.

Kolokolova, L., Jockers, K., Chernova, G., Kiselev, N., Apr. 1997. Properties of Cometary Dust from Color and Polarization. Icarus 126, 351-361.

Krishna Swamy, K. S., 2010. Physics of comets. World Scientific Publishing Co. Pte. Ltd., Singapore.

Mishchenko, M. I., Rosenbush, V. K., Kiselev, N. N., Lupishko, D. F., Tishkovets, V. P., Kaydash, V. G., Belskaya, I. N., Efimov, Y. S., Shakhovskoy, N. M., 2010. Polarimetric Remote Sensing of Solar System Objects. Akademperiodyka, Kyiv.

Öhman, Y., Jun. 1939. On some observations made with a modified Pickering polarigraph. MNRAS 99, 624.

Öhman, Y., 1941. Measurements of polarization in the spectra of comet Cunningham (1940 C) and comet Paraskevopoulos (1941 C). Stockholms Observatoriums Annaler 13, 11.

Rosenbush, V., Ivanova, A., Kiselev, N., Afanasiev, V., Kolesnikov, S., Shakhovskoy, D., Jul. 2014. Linear and circular polarimetry of recent comets: Observational results for eight comets. In: Muinonen, K., Penttilä, A., Granvik, M., Virkki, A., Fedorets, G., Wilkman, O., Kohout, T. (Eds.), Asteroids, Comets, Meteors 2014. p. 450. 